\title{
Virtual Workshops on the road: co-designing with drivers, within context in real-time
}

\author{
Daniel de la Flor Aceituno ${ }^{1}$, Joseph Giacomin ${ }^{1}$, Alessio Malizia ${ }^{2}$ and Lee Skrypchuk ${ }^{3}$, \\ ${ }^{1}$ Human Centred Design Institute, Brunel University, UB8 3PH London, UK \\ \{daniel.delafloraceituno,Joseph.Giacomin\}@brunel.ac.uk \\ ${ }^{2}$ School of Creative Arts, University of Hertfordshire, AL10 9AB Hertfordshire, UK \\ a.malizia@herts.ac.uk \\ ${ }^{3}$ Human Machine Interface Research, Jaguar Land Rover, CV3 4LF Coventry, UK \\ lskrypch@jaguarlandrover.com
}

\begin{abstract}
An investigation was performed of the characteristics of real-time, virtually present, contextual inquiry between automobile drivers and automotive designers. 28 participants were remotely interviewed while they were in one of two contexts: either sitting in a bare isolated meeting room or when operating a driving simulator. Open questions of the type involved in concept design were used as the basis of the real time interview. The interview transcripts were analysed by means of discourse analysis, thematic analysis and evaluations of degree of creativity. The results revealed that the real time automotive interview context provided richer information in terms of the quantity of the words expressed, the variety of the words expressed, and in terms of the judged degree of creativity of the statements.
\end{abstract}

Keywords: human centred design, co-design, design, contextual inquiry, interviewing, discourse analysis, thematic analysis, creativity, driving, automobile, simulator.

\section{Introduction}

The automotive industry is currently facing an era of rapid evolution driven by the development of sophisticated electronics and algorithms, which are affecting user behaviour, and gradually changing customer experience [1]. In order to explore the implications of this evolution, automotive designers currently apply methodologies such as online surveys, telephone interviews and more recently, trend analysis [2][3]. These tend to focus on drivers' opinions, not necessarily capturing how they actually experience the automobiles. In addition, the methods rely on human long-term memory which is affected by a large list of cognitive processes [4]. These processes include the "location updating effect" [5], which refers to a decline in memory when people move from one place to another, and the "fading affect bias" [6], which involves affect associated with negative memories fading faster than affect associated with positive memories. These processes lead to inaccuracies and biases [4].

Aiming to improve the situation, researchers have recently shifted their interest towards Human-Centred Design, which can be considered as a form of structured empathy [7]. In turn, there is an increasing tendency to collaborate with customers [8]. Part of this community focus is on contextual inquiry [9], which in the automotive context refers to collaborating with drivers while they are inside the automobile. This reduces several cognitive biases including the "location updating effect" [5]. 
Most recently, researchers started exploring the possibility of connecting designers with users in real-time while they are driving [10], [11]. Those researchers advocate for the use of the co-design approach in order to provide carmakers with both ethnographic data and creative ideas generated through contextual inquiry [9]. This paper describes a research study which was performed to investigate the possibilities of real-time collaborative dialogue between drivers and designers, and to evaluate the benefits of within context interaction on the quantity and creative qualities of the information which can be gathered.

\section{Method}

\subsection{Real-Time Collaboration Taxonomy}

Participants were interviewed in one of two study contexts. The contexts were when sitting in a bare and isolated meeting room or when using a driving simulator. The interviews were transcribed, and the information was subsequently analysed to explore its usefulness.

Literature in innovation frequently uses the term 'useful' interchangeably with the term 'creative' [12]. Further, literature advocates that the collection of greater amounts of information correlates with the collection of more creative ideas [13]. Therefore, the information collected in this study was considered to be more 'useful' and 'richer' when it involved a greater number of words, a greater variety of words, a greater number of themes, or when it was assessed as more creative by an independent panel of experts. The research hypotheses of the current study were the following:

- Within context interviews provide richer information in terms of word quantity and variety.

- Within context interviews provide richer information in terms of number of themes.

- Within context interviews provide richer information in terms of creativity.

\subsection{Stimulus Materials}

The bare and isolated meeting room. To simulate the typical context in which most interviews and focus groups are held in current practice, a small and bare room (no decoration or items of equipment other than table and seat) of the university was chosen for the activity. The room provided no obvious visual or acoustic texture or distractions.

The driving simulator. The simulator was composed of three elements: a BMW MINI body shell, a computer under the bonnet which processed the within-vehicle systems (i.e. steering wheel, gearbox, pedals, etc.) and a computer installed outside the vehicle which hosted the "XPI DS2 Full Car Simulator" [14] simulation and monitoring software.

The interview. The interview questions were based on the verbal version of the Torrance Test of Creative Thinking [12] and the structure of the interview was designed to prompt a creative dialogue based on the principles of effective interviewing [15]. The subject selected for the creative conversation was the In-Vehicle Navigation System (IVNS) due to it being one of the main sources of distraction inside cars and, hence, a target for possible future improvements [16]. 


\subsection{Participant Recruitment}

The sample size adopted for the study was 28 participants. Fourteen were interviewed through a telephone while sitting in the bare and isolated meeting room, while fourteen were interviewed through a telephone while steering the driving simulator (each participant was interviewed only once to reduce learning effects). Each group of 14 participants consisted of equal numbers of men and women. The participants had an average age of $26(\mathrm{SD}=3)$ with a range of 21 to 36 years old and an average of 6 years of experience driving $(\mathrm{SD}=4)$ with a range of 1 to 18 years holding the driving license.

\subsection{Measurement Instruments}

Discourse Analysis. The objective metrics adopted for the study were the total number of words spoken, the word variety (number of unique words occurring in the transcripts) and the word frequency (number of times that a given word occurred in the transcripts) [17]. The automated tool used to conduct the analysis was "Word Smith Tools 7.0" [18].

Thematic Analysis. The method selected for the thematic analysis was the General Inductive Approach due, to its clarity and effectiveness in exploring repeating topics [19]. The approach leads to the creation of summary themes or categories from the raw data. The aim of the thematic analysis was the selection and categorisation of ideas which could help to improve the IVNS. Four independent coders were selected from different professional backgrounds, age groups and genders to obtain a range of perspectives. The procedure followed an approach outlined by Thomas [19]. The metric used to measure inter-coder consistency was the percentage of agreement in assigning ideas to categories [20].

Creativity Analysis. The Consensual Assessment Technique [12] was used to estimate the degree of creativity of the interview contents. The technique relies on the judgement of a panel of experts for the evaluation of creative achievement. Six professionals were selected for the expert panel. The main criteria used to select the professionals was that of having a $\mathrm{PhD}$ or significant industrial experience in psychology, design or engineering, and a minimum of 3 years of working experience in their respective sector [21].

\section{Results}

\subsection{Discourse Analysis}

Participants who answered the interview questions from the driving simulator provided longer answers in terms of the number of words than those who were interviewed from the bare isolated meeting room $(F=9.830, p=0.004)$. Regarding the variety of vocabulary used, the within context interview of the driving simulator provided more varied vocabulary than the interview from the bare isolated meeting room $(F=7.618, p=0.010)$. The interviews which took place while the respondents were driving the simulator did not however last longer ${ }^{1}$ than the interviews that took place while the participants were located in the bare isolated meeting room $(\mathrm{F}=0.171, \mathrm{p}=0.683)$. Table 1 presents the summary values.

\footnotetext{
${ }^{1}$ Time length refers to the number of seconds between the instant in which the interviewer started asking the first question and the moment in which the respondent stopped answering the last question.
} 
Table 1. Mean and Standard Deviation (SD) values of the number of words, variety of words, and interview time durations determined across the complete set of participants.

\begin{tabular}{cccccc}
\hline & & Word Quantity & Word Variety & Seconds & Minutes \\
\hline \multirow{2}{*}{ Isolated Room } & Mean & 586 & 200 & 575 & $9^{\prime} 35^{\prime \prime}$ \\
& $S D$ & 205 & 43 & 129 & $2^{\prime} 9^{\prime \prime}$ \\
\multirow{2}{*}{ Driving Simulator } & Mean & 880 & 249 & 552 & $9^{\prime} 12^{\prime \prime}$ \\
& $S D$ & 285 & 52 & 164 & $2^{\prime} 44^{\prime \prime}$ \\
\hline
\end{tabular}

In terms of word frequency, the ten nouns which were used most frequently in both contexts were: car, navigation, GPS, time, maps, places, example, Google, road and way.

\subsection{Thematic Analysis}

The coders extracted 40 improvement ideas from the raw data of the transcribed interviews, which were subsequently clustered into three main themes with an inter-coder agreement of 0.9. The three main themes were:

Navigation Instructions. The suggestions which were made in relation to improving how the instructions were given $(n=14)$ ranged from visual issues, such as the use of real pictures of the street, to verbal issues such as the anticipation or the wording used when providing instructions to the driver.

Connectivity \& Software Updates. The proposals made to improve the connectivity of the device to the network $(n=10)$ included the ability to download maps in order to facilitate operation in zones of degraded GPS coverage, and the inclusion of an on-board AI such as Siri or Alexa.

Customised Experience. The ideas in relation to the customising of the user experience $(n=16)$ ranged from passenger monitoring, to suggesting alternative destinations, to tracking nearby friends and notifying the driver about their presence.

Table 2 below presents the number of independent improvement ideas extracted from the transcripts of the interviews performed in each of the two study contexts.

Table 2. Number of improvement ideas extracted by the coders in each context.

\begin{tabular}{lcccc}
\hline & $\begin{array}{c}\text { Isolated } \\
\text { Room }\end{array}$ & $\begin{array}{c}\text { Driving } \\
\text { Simulator }\end{array}$ & $\begin{array}{c}\text { Both } \\
\text { Contexts }\end{array}$ & Total \\
\hline Navigation Instructions & 2 & 5 & 7 & 14 \\
Connectivity \& Software Updates & 1 & 2 & 7 & 10 \\
Customised Experience & 3 & 9 & 4 & 16 \\
\hline
\end{tabular}

\subsection{Creativity Analysis}

Each of the six professional experts was first asked to articulate his or her own personal definition of creativity, and to define criteria for measuring it across a set of statements. The declared self-selected criteria of creativity included the concepts of positive thinking, elaboration, surprise, originality, abstractness and novelty.

Each professional expert was next provided an individually randomised list of the 28 statements which had been provided by the participants in response to the question: "If you were to write a newspaper headline to summarise your experience with navigation system 
what would it be?". The core creative question provided an opportunity for the interviewees to furnish high level conceptual accounts of their experience, leveraging any relevant metaphors which were being used to make sense of the situation.

Each professional expert was asked to organise the 28 statements from the first (most creative) to the last (least creative) using his or her own self-selected criteria. The method of analytic hierarchy process [22] was then used to assign a numerical value to the rank order position of each statement, and to calculate the average score (from 0 to 100) of the statement across the complete ensemble of expert opinions.

A statistically significant difference was found between the creativity scores of the statements made in the bare isolated meeting room with respect to those expressed in the driving context $(\mathrm{F}=7.188 ; \mathrm{p}=0.013)$. The statements made while driving were scored as more creative $(M=59.4 ; \mathrm{SD}=15.5)$ than those expressed in the meeting room $(\mathrm{M}=44.2 ; \mathrm{SD}=$ 14.6). To illustrate the nature of the differences, Table 3 below provides the five statements which were judged to be the most creative, the five which were judged to be the least creative, along with the context in which each statement was made.

Table 3. The five most creative statements, the five least creative statements and the context in which the statement was provided.

\begin{tabular}{cccc}
\hline Answer Provided by the Respondents & Mean Score & $\begin{array}{c}\text { Isolated } \\
\text { Room }\end{array}$ & $\begin{array}{c}\text { Driving } \\
\text { Simulator }\end{array}$ \\
\hline A great power takes a great responsibility & 86.3 & $\mathrm{X}$ & $\mathrm{X}$ \\
Lifesaver & 82.7 & & $\mathrm{X}$ \\
My salvation & 77.4 & & $\mathrm{X}$ \\
Your friendly virtual co-pilot & 73.8 & & $\mathrm{X}$ \\
SAT NAV saves the day! & 71.4 & & \\
\hline Useless navigation & 36.9 & $\mathrm{X}$ & \\
My hard experience with the GPS & 36.3 & $\mathrm{X}$ & \\
Useful but the voice is annoying & 30.4 & $\mathrm{X}$ & $\mathrm{X}$ \\
Not a very intuitive system & 25.0 & $\mathrm{X}$ & \\
Travel guide to getting you from A to B & 29.2 & & $\mathrm{X}$ \\
\hline
\end{tabular}

\section{Discussion}

The results of the all three analysis confirmed the hypotheses that the within context interview provided richer information in terms of word quantity, word variety, and creativity. However, the results of all three analysis performed in the current study rejected the hypothesis that the within context interview provided richer information in terms of the number of themes covered.

The driving simulator context was found to produce an increased number of words and an increased variety of words, on average, with respect to the bare isolated meeting room. The greater number and variety appeared to be attributable to the interviewees providing greater detail in their explanations. Significant differences were not noted in the number of themes which were expressed, but the level of detail and the degree of creativity was greater in the case of the interviews which were performed within the driving context. 


\section{Acknowledgment}

This research was funded and supported by Jaguar Land Rover.

\section{References}

1. Smith, T., Vardhan, H., \& Cherniavsky, L.: Humanising Autonomy. Where are we going. London: USTWO (2017)

2. Seat: Annual Report. Retrieved from https://www.seat.com/company/annualreport/management.html. (2017)

3. Audi: Annual Report. Retrieved from https://www.audi.com/en/company/investor-relations/reportsand-key-figures/annual-reports.html (2017)

4. Benson, B.: Cognitive bias cheat sheet. Better Humans. (2016)

5. Radvansky, G. A., Krawietz, S. A., \& Tamplin, A. K.: Walking through doorways causes forgetting: Further explorations. The Quarterly Journal of Experimental Psychology. 1632-1645. (2011)

6. Walker, W. R., \& Skowronski, J. J.: The fading affect bias: But what the hell is it for? Applied Cognitive Psychology, 23(8), 1122-1136. (2009).

7. Giacomin, J.: What is human centred design?. The Design Journal. 17(4) 606-623. (2014)

8. Ramm, S., Giacomin, J., Malizia, A., \& Anyasodo, B.: Using participatory design workshops to identify what feels natural about using a car's secondary controls. ATA - Ingenieria dell'Autoveicolo, 68 46-52. (2015)

9. Gkatzidou, V., Giacomin, J., \& Skrypchuk, L.: Automotive Habitat Laboratory: a facility for automotive co-design. Proceedings of the 7th International Conference on Applied Human Factors and Ergonomics. Orlando, Florida, USA (2016)

10.Martelaro, N., \& Ju, W.: WoZ Way: Enabling Real-time Remote Interaction Prototyping \& Observation in On-road. CSCW '17 Proceedings of the 2017 ACM Conference on Computer Supported Cooperative Work and Social Computing (pp. 169-182). Portland, Oregon, USA: ACM. (2017)

11.Giuliano, L., Germak, C., \& Giacomin, J.: Effect of Driving Context on Design Dialogue. International Conference on Applied Human Factors and Ergonomics: Advances in Ergonomics in Design. 134-144. Orlando, Florida, USA (2017)

12.Said-Metwaly, S., Noortgate, W. V., \& Kyndt, E.: Approaches to Measuring Creativity: A Systematic Literature Review. Creativity: Theories, Research, Applications, 4(2), 238-275. (2017)

13.Pedersen, T.: Recipe for Creativity: Crank Out Ideas and Step Away. PsychCentral. (2019)

14.The Driving Simulator Company, http://www.xpisimulation.com/products/full-car-simulator

15.Strachan, D. Making Questions Work: A guide to What and How to Ask for Facilitators, Consultants, Managers, Coaches, and Educators. Wiley. (2007)

16.Lee, B., Lee, Y. J. N., Park, S., Kim, H., Lee, S. J., \& Kim, J.: Driver's distraction and understandability (EOU) change due to the level of abstractness and modality of GPS navigation information during driving. Procedia computer science, 39, 115-122. (2014)

17.Baker, P.: Using corpora in discourse analysis. London: Continuum International Publishing Group. (2006)

18. Word Smith Tools, https://www.lexically.net/wordsmith/

19.Thomas, D. R.: A General Inductive Approach for Analyzing Qualitative Evaluation Data. American Journal of Evaluation, 27(2), 237-246. (2006)

20.Lombard, M., Snyder-Duch, J., \& Bracken, C. C.: Content Analysis in Mass Communication: Assessment and Reporting of Intercoder Reliability. Human Communication Research, 28(4), 587604. (2006)

21.Origgi, G. Reputation: What It Is and Why It Matters. Oxfordshire, UK: Princeton University Press. (2018)

22.Saaty, T.L.: Decision making with the analytic hierarchy process. Int. J. Services Sciencesm 1(1), 83,98. (2008) 